\section{Reaching out}

\section{Katherine Mack, Karl Kruszelnicki, Lisa Randall, Jessica Wade, Jim Al-Khalili and Vlatko Vedral}

In the midst of the COVID-19 pandemic, science is crucial to inform public policy. At the same time, mistrust of scientists and misinformation about scientific facts are rampant. Six scientists, actively involved in outreach, reflect on how to build a better understanding and trust of science.

\section{Show the world who we are}

Katie Mack. It's a common refrain these days that scientists aren't trusted, experts aren't valued and science is being ignored. That's certainly evident in areas of national leadership in many countries, and a few issues that should be scientific ones seem to have been placed in the category of partisan dogma, rather than debatable policy. But when it comes to how we, as scientists, are viewed by the general public, poll after poll ${ }^{1,2}$ finds that scientists are, largely, trusted to talk about science - more trusted than journalists, business leaders or elected officials. However, polls also show that our motives are considered suspect. Scientists are frequently perceived as less caring or moral than non-scientists ${ }^{3}$, and general opinion is split as to whether we should be allowed to weigh in on matters of policy ${ }^{1}$. I suspect that this ties into another disheartening phenomenon: most Americans (for instance) can't name a living scientist and don't know what we actually do.

There are nuances to all of this, but from my perspective, we have an opportunity to make ourselves better heard and to engage more responsibly, just by being more visible and open with the public. Let everyone know what we do, how we come to our conclusions and what drives us. Listen more. Engage respectfully. I don't think scientists should have the last say in every policy decision, but if we can let non-scientists understand who we are and how we can help, we might find ourselves more often invited to the table and better able to contribute when we're there.

\section{Tell a good story}

Karl Kruszelnicki. What I absolutely love about scientists is that they generally don't mind admitting when they are wrong.
Good science means accepting new data or better explanations - even if that means what you used to believe is outdated. It's more than OK to say you don't know. I'm pretty sure I say "I don't know" at least once each week! It's not a weakness. It means there's something new for you to learn.

A good example of this came out of one of my radio shows. A listener was looking at rain puddles. He wanted to know, "How come, no matter which way the wind blows, the individual raindrops make ripples in the puddles that form circles, not ellipses?" I was happy to tell him I would treat the question as my homework - because I didn't know the answer right away.

At morning tea, I asked a whole table of physics professors my homework question. There was a broad range of 'robust' opinion and conjecture. But then one of them said, "All the raindrop does is give the puddle its initial energy, and that can come from any direction and will create a slightly elliptical disturbance. But after that, the speed at which the energy propagates depends only on the physical properties of the water. It will travel at constant speed, giving a basically circular expanding ripple." Suddenly the other professors dropped their own pet theories, saying, "Your answer works best. I was wrong. You're right." There were no hard feelings, just a mutual sense of pleasure that the problem was solved.

But here's the paradox. This readiness to change exposes science to mistrust. The public isn't used to this kind of professional openness. We live in a world of sound bites. A 15-second clip is not enough to properly explain a complicated subject and discuss all the pros and cons. The idea that something is still being developed, or that there is not unanimous agreement, is often seen as unreliability, rather than honesty.

Getting the message out that science doesn't pretend to know everything, and that it will happily self-correct when it finds a better solution, is really important. My way of doing that is to wrap the facts into a good story - something relatable with a catchy intro, a deeper middle section and, ideally, a funny ending!

\section{Tell the truth}

Lisa Randall. As people worldwide have the rug pulled out from under them as the world radically transforms - at least for the time being - we are all faced with the question of how to move forwards and how to identify our role in surviving this mess. The role of physical scientists is less obvious than that of doctors, biologists and chemists, who have a chance to directly contribute to the health of both individuals and society as a whole, as they search for cures, preventive measures or simply palliative care. Physics does, however, play a role in medical procedures. Diagnostic techniques like X-rays and MRI, as well as physical breathing devices (ventilators), all stem from advances in physics. But the role of physicists might be less obvious.

First and foremost, all scientists have an obligation to tell the truth. Politicians, policymakers, economists, churches, psychologists and artists can all take information and interpret it for public consumption. But the public have a desperate need for facts - facts based on the increasingly extensive statistics about the progression of the disease - both in individuals and in society.

And with human lives at stake, scientists have an obligation to get the information out and to find ways to make people listen. Unfortunately, the biggest mouthpieces don't always belong to the wisest people or those with the best understanding. But scientists (and others) should use whatever mouthpiece is at their disposal be it social media, blogs or journals such as Nature Reviews Physics.

Difficult as it may be, we scientists must use our influence to support those dealing with the current pandemic and try to convince the people in charge to listen and act according to the best scientific and 
societal advice. We have to stand more strongly and more vocally behind scientific advisers who are trying to take a stand in the face of inexplicable resistance. Science is only one part of the many societal issues we are now up against, but it's an important one and one that can provide the most reliable information to be absorbed into workable, sensible policies. We should remember that the scientific community is far-reaching and extends not only into the research and medical community but into a good fraction of the economy, too. People want answers - at a rate faster than we can find them. But they also want to hear trustworthy information from people who are not afraid to tell the truth. Even in the face of the challenges ahead, the scientific community should stand strong and continue to vocalize its knowledge.

\section{Tackle misinformation}

Jess Wade. There's no doubt that science and engineering play an important role in combatting the ongoing pandemic. Whether it's the biochemistry laboratories that are racing to create vaccines and antibody tests, the epidemiologists who are helping governments to write new policies or the makerspaces and workshops that are mass-producing personal protective equipment and ventilators, the world is more dependent on the innovations of academia and industry than ever before. What's less clear is the role of physical scientists: how the scientists who can't run an enzyme-linked immunosorbent assay (ELISA) or predict the spread of a viral outbreak can be useful during these uncertain times.

We physicists can spend our time away from the lab bench trying to tackle misinformation. At a time so dependent on the insight and experience of experts, pseudoscience is threatening the global response. From race myths to $5 \mathrm{G}$ fears and dangerous drug advice, social media are accelerating panic and confusion. So much so that Google recently announced a US\$6.5 million fund to support fact-checking groups ${ }^{4}$. COVID-19 is the first pandemic of the preprint era; science is happening quickly, it is being reported quickly and the overwhelming amount of technical information is often difficult to process. That's where we can step in: we can all edit Wikipedia. Wikipedia is currently more important than ever before: it's non-partisan, it's simple to understand, it's available offline, it's educational (particularly valuable for half of the

\section{The contributors}

Katie Mack is a theoretical astrophysicist exploring a range of questions in cosmology, the study of the universe from beginning to end. She is currently an assistant professor of physics at North Carolina State University. Her first popular book, The End of Everything (Astrophysically Speaking), will be out in August. She can be found on Twitter as @AstroKatie.

Karl Kruszelnicki is a science generalist, with an enthusiastic public following in Australia. He has frontlined in multiple media for decades. He is writing his 46th book and does half a dozen science QEA radio shows every week. He is a Fellow in the School of Physics at the University of Sydney. He has degrees in maths and physics, biomedical engineering, medicine and surgery. He can be found on Twitter as @doctorkarl.

Lisa Randall studies theoretical particle physics and cosmology at Harvard University. Her research connects theoretical insights to puzzles in our current understanding of the properties and interactions of matter. Additionally, she engages with the public through her popular science books, articles, lectures, and radio and TV appearances.

Jess Wade is an excitable scientist with an enthusiasm for equality. By day, she is based in the Department of Chemistry at Imperial College London, where she creates superthin films out of organic electronic materials that emit and absorb circularly polarized light. She spends her evenings editing Wikipedia, working to make the internet less sexist and racist. She can be found on Twitter as @jesswade.

Jim Al-Khalili, FRS, is a theoretical physicist, author and broadcaster. He holds a Distinguished Chair in physics at the University of Surrey, where he teaches and conducts his research in nuclear physics and open quantum systems. As well as his popular science writing, he is a regular presenter on TV and hosts the long-running BBC Radio 4 programme, The Life Scientific. His latest book, The World According to Physics, is out now. He can be found on Twitter as @jimalkhalili.

Vlatko Vedral is a professor of physics at Oxford and National University of Singapore working on quantum physics. He has received many awards for his work, including the Royal Society Wolfson Research Merit Award and the World Scientific Medal and Prize, and was elected a Fellow of the Institute of Physics in 2017. He gives regular interviews to the media and has written articles for New Scientist, Scientific American and other major newspapers, as well as two popular science books.

global population who are currently homeschooling) and it's multilingual. Editing isn't complicated - if you've sent an e-mail or submitted a manuscript to Nature Reviews Physics, you're more than capable enough to contribute - and you can write about anyone or anything that fascinates you. So instead of scheduling another Zoom meeting, why not click 'edit' and join a network of volunteers helping to make the internet better.

\section{Choose doubt over certainty}

Jim Al-Khalili. In our complex modern world of conflicting ideologies, opinions are becoming ever more polarized and entrenched. Many people today seem increasingly prepared to believe what they hear and read if it reinforces their existing biases, while dismissing any opposing arguments. Of course, there have been cases in which scientists have behaved in a similarly dogmatic way, but the scientific method encourages self-criticism. Compare a scientific theory with a conspiracy theory: advocates of the latter would point out that, like any good scientist, they too are sceptics and rationalists who value the importance of evidence. But at its heart, a conspiracy theory is the polar opposite of a scientific one, in that its proponents will seek to assimilate whatever evidence there is against it and interpret it in a way that confirms rather than repudiates their core idea, thus, making it unfalsifiable.

With so many unscientific notions spreading across the internet, particularly during the COVID-19 pandemic, I feel strongly that we, as scientists, must stress the unsung virtues of the scientific method, such as maintaining an open mind and being prepared to modify our views when we have a deeper understanding of a subject or in the light of new data or observations. One feature that many in wider society see wrongly as a weakness is the way scientists value the importance of doubt. Being inflexible about what we believe to be true in science means that our views are no different from an ideological belief. But scientific progress is not made that way. Always maintaining a level of doubt, even about our most cherished scientific theories, is a consequence of our desire to seek the truth, rather than to simply win an argument.

This attitude is crucial during the current pandemic. When politicians say they are following the best available scientific evidence, then, provided they are being honest about it, that is the best we can hope for. 


\section{VIEWPOINT}

Society must accept that, sometimes, a shift in approach is necessary as more data are accumulated and model predictions become more reliable. The message we scientists must get across is that, sometimes, changing your mind doesn't mean you are confused or uninformed, but, rather, that you have a better understanding than you did before.

\section{Share the spirit of science}

Vlatko Vedral. Science has undeniably greatly improved the human condition through various technological innovations and, yet, it seems to me that scientists are still largely distrusted by the public. I propose that this is because science, despite all its successes, has not managed to properly cater to the 'human soul'.

This, I believe, is an unfortunate outcome of how we teach science in schools and how we scientists communicate it to the general public. When the cultural value of science is debated, we scientists frequently emphasize the 'nothing-but' message of science. A human being is nothing but a bag of molecules. Thoughts and emotions are nothing but a bunch of chemical reactions. The universe is nothing but a handful of mathematical laws. If this really was the main message of science, I doubt that any child would ever want to grow up to become a professional scientist.

This is a pity, especially because the spirit of science is the exact opposite of the 'nothing-but'. Rather, science embodies the 'not-that' philosophy, and it would behove us scientists to communicate this more widely and effectively. Where does the universe come from? We don't know, but it was not created by a giant turtle. What is gravity? We don't know, but it does not require angels to push planets around the sun. Science is driven by a sense of wonder. The explanations it gives of natural phenomena, which are made by placing limitations on what can be said and done, seem, to me, always more creative and magical than the 'nothing-but' view would suggest. Of course there are rules. Nothing can travel faster than light. Nothing can tell gravity apart from acceleration. And nothing can violate Heisenberg's uncertainty principle. The rest, as far as physics is concerned, is limited by nothing but our human imagination.

Katherine $\operatorname{Mack}^{1}{ }^{\bowtie}$, Karl Kruszelnicki ${ }^{\square}$ Lisa Randall $^{3}$, Jessica Wade ${ }^{4 凶}$, Jim Al-Khalili ${ }^{\square}$ and Vlatko Vedral ${ }^{6}$

'Department of Physics, North Carolina State University, Raleigh, NC, USA.
${ }^{2}$ School of Physics, University of Sydney, Sydney, Australia.

${ }^{3}$ Department of Physics, Harvard University, Cambridge, MA, USA.

${ }^{4}$ Department of Chemistry, Imperial College London, London, UK.

${ }^{5}$ Department of Physics, University of Surrey, Guildford, UK.

${ }^{6}$ Department of Physics, University of Oxford, Oxford, UK.

凶e-mail:kmack@ncsu.edu; drkarl@sydney.edu.au; randall@g.harvard.edu; jessica.wade@imperial.ac.uk; j.al-khalili@surrey.ac.uk; vlatko.vedral@physics.ox. ac.uk

https://doi.org/10.1038/s42254-020-0185-5

Published online 18 May 2020

1. Funk, C. Key findings about Americans' confidence in science and their views on scientists' role in society. Pew Research Center https://www. pewresearch.org/ fact-tank/2020/02/12/key-findings-about-americansconfidence-in-science-and-their-views-on-scientistsrole-in-society/ (2020).

2. $3 \mathrm{M}$. 3M State of Science Index: 2018 Global Report. 3M https://multimedia.3m.com/mws/media/15152950/ presentation-3m-state-of-science-index-2018-globalreport-pdf.pdf (2018)

3. Rutjens, B. T \& Heine S. J. The immoral landscape? Scientists are associated with violations of morality PLOS ONE 11, e0152798 (2016).

4. Mantzarlis, A. COVID-19: \$6.5 million to help fight coronavirus misinformation. Google https://blog. google/outreach-initiatives/google-news-initiative/ covid-19-65-million-help-fight-coronavirusmisinformation/ (2020).

Competing interests

The authors declare no competing interests.

(C) Springer Nature Limited 2020 